Article

\title{
Online State of Health Estimation for Lithium-Ion Batteries Based on Support Vector Machine
}

\author{
Zheng Chen (1), Mengmeng Sun, Xing Shu, Renxin Xiao and Jiangwei Shen * \\ Faculty of Transportation Engineering, Kunming University of Science and Technology, Kunming 650500, China; \\ chen@kmust.edu.cn (Z.C.); smm1023@foxmail.com (M.S.); shuxing92@163.com (X.S.); \\ xrx1127@foxmail.com (R.X.) \\ * Correspondence: shenjiangwei6@163.com; Tel.: +86-13888050191
}

Received: 8 April 2018; Accepted: 31 May 2018; Published: 4 June 2018

\begin{abstract}
In this paper, a novel state of health $(\mathrm{SOH})$ estimation method based on partial charge voltage and current data is proposed. The extraction of feature variables, which are energy signal, the Ah-throughput, and the charge duration, is discussed and analyzed. The support vector machine (SVM) with radial basis function (RBF) as kernel function is applied for the $\mathrm{SOH}$ estimation. The predictive performance of the SOH by the SVM are performed with full and partial charging data. Experiment results show that the addressed approach enables estimating the $\mathrm{SOH}$ accurately for practical application.
\end{abstract}

Keywords: capacity degradation; charge voltage; state of health (SOH); support vector machine (SVM)

\section{Introduction}

Nowadays, oil crisis and environmental pollution bring increasing concern to our daily life. Vehicle electrification can effectively mitigate their influences. Among all the solutions, the most popular manner is electric vehicles (EVs), which employ the energy storage system and one or two motors to power the vehicle [1-3]. In an EV, hundreds to thousands of cells connected in series/parallel topologies enable powering the EV for the maximum range of $100-500 \mathrm{~km}$. Consequently, it is necessary to manage the battery pack to ensure safe and proper operations by a so-called battery management system (BMS). With increase of cycle numbers of lithium-ion batteries, the electrode materials gradually become inactive, leading to the performance degradation of the battery. The battery state of health $(\mathrm{SOH})$ is an estimation to evaluate the battery capability status, by which the battery related inner parameters including state of charge (SOC) and remaining driving range can be accessed with higher precision. Actually, the battery aging rate depends on the battery operation state, including charging and discharging rates, depth of discharge, and the external environment such as ambient temperature and storage conditions. Gaining more knowledge about battery aging can potentially bring improvement of the battery life and avoid occurrence of safety hazard [4-6], and thus it motivates researchers to conduct the $\mathrm{SOH}$ estimation for lithium-ion batteries.

Currently, various studies on the $\mathrm{SOH}$ estimation have been put forward $[7,8]$, which can be classified into the physical-based method, the parameter variation method of the equivalent circuit model (ECM), the data-driven method and the fusion method [9]. The physical-based method considers the physical degradation of the battery and includes the electro-chemical based model and external characteristic model. The model based electro-chemical property is extensively investigated for the SOH estimation $[10,11]$. The electro-chemical based methods establish the model based on the internal specific physical and chemical reaction of the battery during the charge and discharge process. However, the complex modeling process and excessive computation intensity limit its wide application. An external characteristic model was introduced [12] to discover the relationship among capacity, 
charge and discharge current rate, discharge depth and temperature, and was validated to be suitable under fixed battery operation conditions. The ECM is a widely employed method for the $\mathrm{SOH}$ estimation [13]. In [13], the Thévenin ECM and the genetic algorithm (GA) are implemented together to identify the parameter variation, and the results show that it can achieve reliable $\mathrm{SOH}$ estimation. To sum up, for physical-based approaches and the ECM based method, various complex equations with considerable parameters are required for the accurate $\mathrm{SOH}$ prediction, and thus it is difficult to achieve the online prediction due to complex computation. The data-driven approach is an efficient and effective method [14-16], which typically includes neural networks (NN), support vector machines (SVMs), Gaussian regression process (GRS), relevance vector machine (RVM), autoregressive and moving average (ARMA), etc. [17-21]. In [16], a SOH estimation method is proposed based on the ARMA model. Liu, D et al. [21] predicted battery degradation trend using GRS model. Considering the possibility analysis, the Dempster-Shafer theory (DST) and the Bayesian Monte Carlo (BMC) method are integrated together to realize battery capacity forecasting [22]. However, these models can only be applied in fixed discharging and charging operations, implying that they are inadequate for real-time application. To conduct better performance for $\mathrm{SOH}$ prediction, fusion methods attract much attention. They employ two or more approaches to operate together thereby achieving the estimation, e.g., ARMA, NN, fuzzy logic, etc. [23]. Although these blended methods can increase the predictive precision to some extent, their excessive complexity brings the heavy computation intensity for practical application.

Aiming to overcome the aforementioned problems, an SVM is introduced to achieve precise $\mathrm{SOH}$ estimation in this paper [24-26]. Different from other data-driven methods, the SVM method is more suitable for nonlinear problems with small samples, and can effectively prevent against local minimization. Moreover, it is not sensitive to dimension of the data and variability. In [24], a SVM classification and regression model is proposed for different status of batteries, and is declared to achieve desirable results. However, the discharging process is infeasible to extract input vectors for on-board $\mathrm{SOH}$ estimation due to stochastic characteristic of the discharge current. In [25], combined with the rain flow theory, the SVM algorithm is applied to predict the $\mathrm{SOH}$ under the complex operating conditions. However, some feature vectors are difficult to measure and calculate in practical application. Therefore, it is imperative to select suitable feature vectors to achieve real-time estimation. In addition, SVM, as a data-driven method, needs large quantity of data to build and train the model, which brings pressure of storage amount for the BMS. From this point, the SVM based SOH estimation algorithm should be carefully designed considering fewer data while achieving high accuracy. This is the main research focus of this paper.

In this paper, the extraction of characteristics variables for the battery $\mathrm{SOH}$ estimation is introduced in detail, where partial charging voltage curve is analyzed and discussed for different aging status [27]. Then, the SVM model with the radial basis function (RBF) as the kernel function is established for the $\mathrm{SOH}$ estimation based on the partial charging voltage data. Experiment validation proves the feasibility of the proposed algorithm. The remainder of this paper is organized as follows. In Section 2, the battery charging process is detailed and analyzed, based on which the related characteristic variables are extracted in depth for the following study. Section 3 introduces the SVM algorithm comprehensively as well as its application for the $\mathrm{SOH}$ estimation. Based on the proposed method, a series of results regarding $\mathrm{SOH}$ estimation are shown and analyzed in Section 4, and key conclusions are summarized in Section 5.

\section{Definition and Description of State of Health}

In this paper, the $\mathrm{SOH}$ of the lithium-ion battery is described by the loss of rated capacity, which can be furnished as,

$$
S O H=\frac{C_{b a t}}{C_{\text {nom }}} \times 100 \%,
$$

where $C_{n o m}$ and $C_{b a t}$ denote the nominal and actual battery capacity, respectively. 
For the sake of accurate $\mathrm{SOH}$ estimation, the extraction of characteristic variables is pivotal. Due to complexity of the battery inner structure and materials, it is difficult to extract efficient features from the data under a static condition. Based on this, data from charge/discharge test are more feasible for the $\mathrm{SOH}$ study. The offline discharge test is a common method for the measure of battery capacity, in which the battery is discharged by a constant rate at a certain temperature; however, this method would accelerate the battery degradation and its whole test time is relatively long. In addition, focusing on on-road application, the lithium-ion battery pack in EVs is irregularly discharged under different driving conditions, leading to difficulty estimating the $\mathrm{SOH}$ online. Instead, the charging process is more applicable for the $\mathrm{SOH}$ estimation, owing to its constant current (CC), as shown in Figure 1. Moreover, in practice, when EVs are charged, the battery charging data are easy to acquire and the BMS has enough capability to handle the $\mathrm{SOH}$ estimation. Considering this, the data during the $\mathrm{CC}$ charge process are selected for the $\mathrm{SOH}$ estimation.

In this paper, the battery cycling data are collected from a public source [28], which records the voltage, current, temperature and capacity for each charge and discharge cycle [28,29]. After screening all the operation process, the charging data of two batteries (called Cell 1 and Cell 2 hereinafter) by the CC method are analyzed and main parameters are listed in Table 1 . They are 18,650-size lithium-ion cells with $2.0 \mathrm{Ah}$ nominal capacity, and $\mathrm{LiNi}_{0.8} \mathrm{Co}_{0.15} \mathrm{Al}_{0.05} \mathrm{O}_{2}$ was applied as the main positive electrode material. The battery is charged under the CC mode first until the battery voltage reaches $4.2 \mathrm{~V}$, and then sustains under the $\mathrm{CV}$ mode until the charging current drops to $20 \mathrm{~mA}$.

Table 1. List of batteries with their operating parameters.

\begin{tabular}{ccccc}
\hline Battery Number & Charge Current (A) & Discharge Current (A) & Ending Discharge Voltage (V) & Temperature $\left({ }^{\circ} \mathrm{C}\right)$ \\
\hline Cell 1 & 1.5 & 2 & 2.7 & 24 \\
Cell 2 & 1.5 & 2 & 2.2 & 24 \\
\hline
\end{tabular}

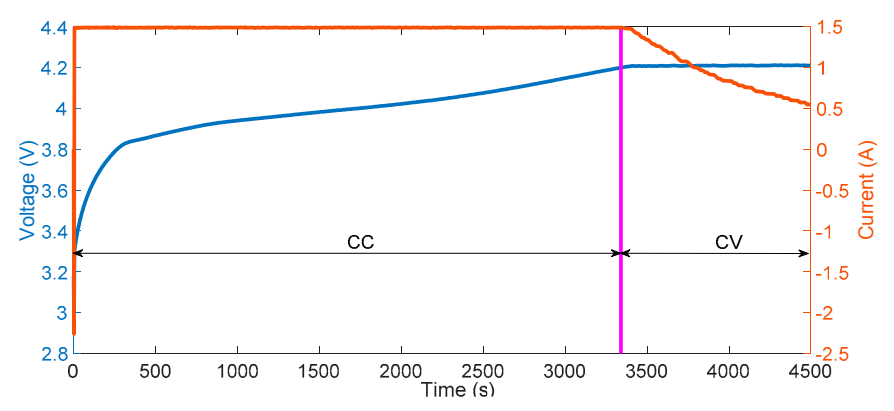

Figure 1. Charge process of battery.

The capacity and the internal resistance are commonly selected to characterize the lithium-ion battery degradation. Actually, the battery internal parameters vary slowly with increase of cycle numbers. The external measured signals can be affected by variation of internal parameters, and thus the shape of externally measured voltage curve would also be changed. Based on this, we can employ the regular charge pattern variation of external signals to depict the variation of $\mathrm{SOH}$. The battery partial voltage variation profile under the CC mode with different cycle numbers is shown in Figure 2, based on which we can find that the tendency of three charge curves are obviously different. From this point, we can extract proper vectors from the CC charging voltage curve to estimate the $\mathrm{SOH}$.

However, when the batteries in EVs are charged for daily use, the initial SOC is rarely the same due to random driving conditions. Moreover, to achieve a uniform $\mathrm{SOH}$ estimation based on the charging data, the data region should be defined properly. In addition, the BMS needs to store and process large data for the $\mathrm{SOH}$ estimation. Thus, we can say that the prerequisite of selecting all CC charging data for the $\mathrm{SOH}$ estimation cannot make sense. 
Here, a novel $\mathrm{SOH}$ estimation approach based on a reasonable voltage region $\left[V_{l}, V_{h}\right]$ during the charging process may be helpful to perform the $\mathrm{SOH}$ estimation, where $V_{l}$ and $V_{h}$ denote the lower and upper limits of the voltage region, respectively. In order to select $V_{l}$ and $V_{h}$, three criterias should be considered. Firstly, the voltage curve starting from the low voltage $V_{l}$ to the end voltage $V_{h}$ at different cycle numbers can reflect the battery aging rate. As shown in Figure 3 , the curves for two different voltage regions $[3.8,4.2]$ and $[3.9,4.0]$ at different cycle numbers are described. We can find that shape of voltage curves with different cycle numbers varies with the selected region. It means that the different charging voltage region curve can reflect the different variation trend of $\mathrm{SOH}$. Secondly, the low voltage $V_{l}$ should be as high as possible. If the charging voltage in the beginning is higher than the selected low voltage $V_{l}$, it is difficult to guarantee the data's effectiveness and to achieve the $\mathrm{SOH}$ estimation. Thirdly, the range of the voltage region $\left[V_{l}, V_{h}\right]$ should be as narrow as possible to reduce data storage capacity and the computation labor.

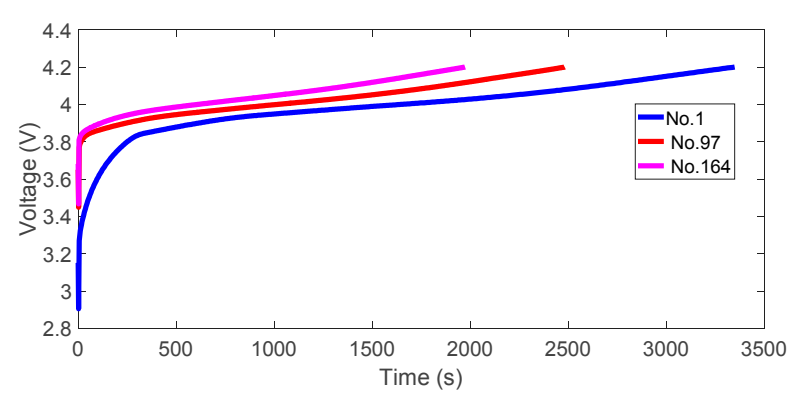

Figure 2. Terminal voltage curves under different battery cycle numbers.

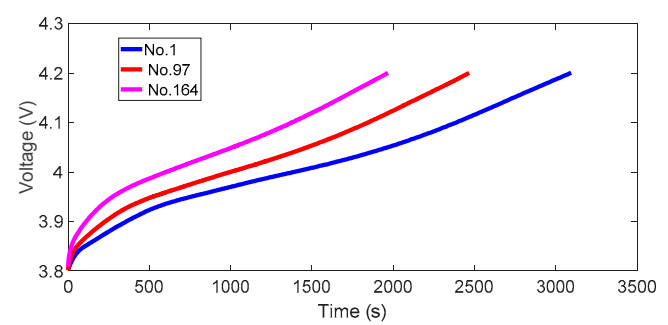

(a)

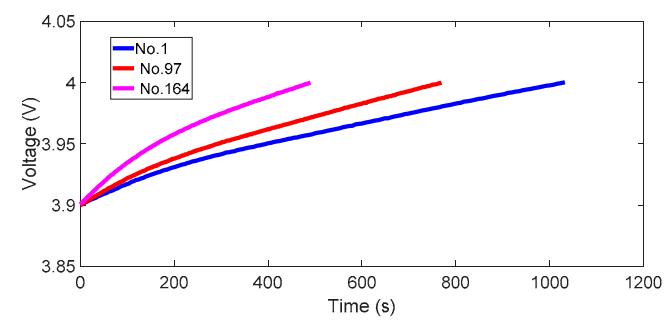

(b)

Figure 3. Terminal voltage curves during battery cycle numbers for different voltage region. (a) The curve of constant current (CC) charging voltage region [3.8, 4.2]; (b) The curve of CC charging voltage region $[3.9,4.0]$.

\section{State of Health Estimation Model}

In this paper, the SVM algorithm is adopted for the $\mathrm{SOH}$ estimation based on the partial voltage variation, which can solve nonlinear regression and irregularity problems. First, the SVM algorithm is introduced along with its regression characteristics. Then, the operation steps of the $\mathrm{SOH}$ estimation are presented based on the calculation process.

\subsection{Support Vector Machine}

Support vector machines, steming from the statistical learning theory, is widely applied for classification and forecast $[23,30]$, which supplies an efficient manner to deal with nonlinear and high-dimensional problems. Here, the input-output dataset regarding the battery CC charging data $\left(x_{i}, y_{i} ; i=1,2, \ldots, l\right)$ is defined, where $x_{i} \in R^{d}$ is d-dimensional input vectors and $y_{i} \in R$ is $\mathrm{SOH}$. For the nonlinear $\varepsilon$-support vector regression ( $\varepsilon$-SVR), the optimization goal is to find a function $f(x)=w^{T} \phi(x)+b$ that tolerates the deviation of $\varepsilon$ with the true value of sample $y$ in a high dimensional feature space, where $\mathrm{w}$ is the weight vector and $\mathrm{b}$ denotes the regression parameter, and $\phi(x)$ maps $\mathrm{x}$ into a high 
dimensional feature space. This can be realized by introducing the error penalty factor $C$ and the slack variables $\xi_{i}$ and $\xi_{i}^{*}$. The related nonlinear problem can be formulated as,

$$
\begin{array}{cc}
\min _{w, b, \xi_{i}, \xi_{i}^{*}} & \frac{1}{2}\left\|w^{2}\right\|+C \sum_{i=1}^{l}\left(\xi_{i}+\xi_{i}^{*}\right) \\
\text { s.t. } & (w \cdot \phi(x))+b-y_{i} \leq \varepsilon+\xi_{i} \\
& y_{i}-(w \cdot \phi(x))-b \leq \varepsilon+\xi_{i}^{*} \\
& \xi_{i}, \xi_{i}^{*} \geq 0, i=1,2, \ldots l .
\end{array}
$$

Then, Lagrange multipliers $\alpha_{i}$ and $\alpha_{i}^{*}$ are employed for solving $\mathrm{w}$ and $\mathrm{b}$, respectively. Finally, the approximating function for predicting the battery $\mathrm{SOH}$ can be gained as,

$$
f(x)=\sum_{i=1}^{n}\left(\alpha_{i}-\alpha_{i}^{*}\right) K\left(x, x_{i}\right)+b,
$$

where $K\left(x, x_{i}\right)=\left(\phi\left(x_{i}\right) \cdot \phi(x)\right)$ is the kernel function. Based on the above offline training, Equation (3) can be programmed in the BMS and then can be applied to achieve the on-board $\mathrm{SOH}$ estimation for real operation. The authors aim to further exploit the potential SVM algorithm applied for the battery $\mathrm{SOH}$ estimation. The primary advantage of SVM is that the support vectors (SVs) can determine the regression model, of which the Lagrange multipliers should satisfy $\alpha_{i} \neq 0$ and $\alpha_{i}^{*} \neq 0$. Support vectors can not only help to catch the significant samples, but also can avoid the so-called curse of dimension. In the next step, the selection of input vectors and the corresponding parameters are conducted to achieve the SVM application.

\subsection{Feature Extraction and Data Processing}

Generally, the steps of building a SVM model contains feature extraction, data processing, optimal SVM parameters searching, data training, data prediction, etc.

Feature variables selection is the key step for training the SVM model. The selected features should reflect the change of voltage curve for different cycle numbers, as discussed in Section 2. In [31], the lithium-ion battery life model is proposed which can be formulated as,

$$
Q_{\text {loss }}=B \cdot \exp \left(\frac{-E_{a}}{R T}\right)\left(A_{h}\right)^{z},
$$

where $B$ denotes the pre-exponential factor, $R$ is the gas constant, $z$ is the power law factor and $E_{a}$ is the activation energy in $\mathrm{J} \mathrm{mol}-\mathrm{i}$ and $\mathrm{T}$ is the absolute temperature. $A_{h}$ is the $A_{h}$-throughput and can present different $C$-rates when charging the battery. In order to quantify the capacity fading behavior with same $\mathrm{C}$-rate, a variable, i.e., time $\mathrm{t}$ during the cycle is introduced. Hence, we can see the loss capacity $Q_{\text {loss }}$ is related with $A_{h}$, and $t$. In addition, energy signal $E_{s}$ can effectively reflect the difference of voltage curve for different $\mathrm{SOH}$ [24]. To sum up, we consider three feature values, i.e., $E_{s}, A_{h}$, and $t$, that need to be extracted from the $\mathrm{CC}$ charging data to track the $\mathrm{SOH}$ variation, which can be yielded as,

$$
\left\{\begin{array}{l}
E_{s}=\int_{t_{1}}^{t_{2}} V^{2} d t \\
A_{h}=\int_{t_{1}}^{t_{2}} I d t \\
t=t_{2}-t_{1}
\end{array},\right.
$$

where $V$ is the battery voltage, $I$ is the battery current, and $t_{1}$ and $t_{2}$ are time nodes when battery voltage reaches $V_{l}$ and $V_{h}$, respectively. Figure 4 describes the variation of these three features across cycles for Cell 2 under different voltage regions. We can observe that, with increase of cycle numbers, the variation trend of these three features is similar. Aiming to easily train the SVM model, the normalization process is imposed in this paper, as shown in Table 2. 
Table 2. An example of feature vectors.

\begin{tabular}{ccccc}
\hline Vector & SOH & $\boldsymbol{t}$ & $\boldsymbol{A}_{\boldsymbol{h}}$ & $\boldsymbol{E}_{\boldsymbol{s}}$ \\
\hline Unscaled & $92.82 \%$ & 3027 & 1.2687 & 48777 \\
Scaled & 1 & 0.7808 & 0.7780 & 0.7932 \\
\hline \multicolumn{5}{c}{ SOH }
\end{tabular}

SOH: state of health.

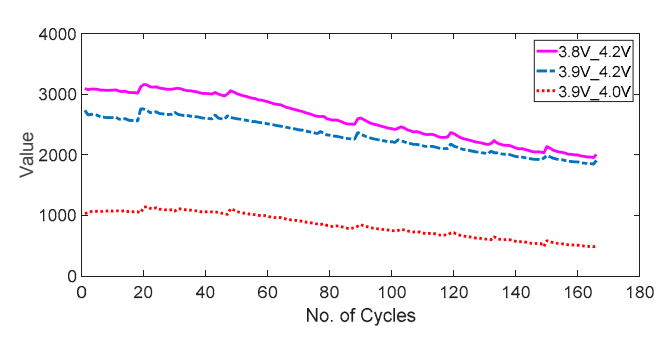

(a)

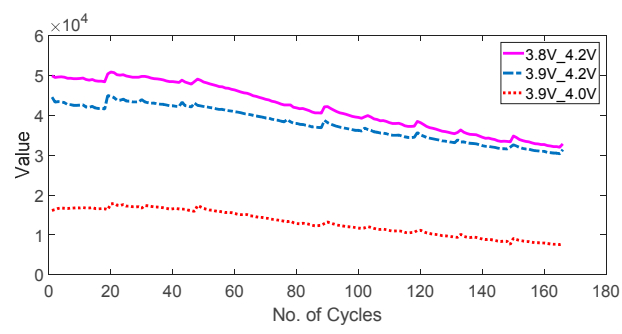

(b)

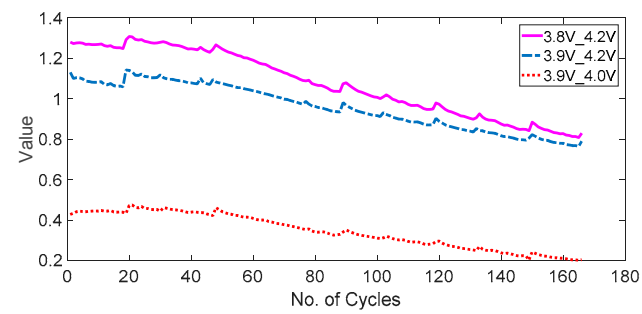

(c)

Figure 4. Feature parameters computed for Cell 2 under different voltage region. (a) The vector of $t$ under different voltage region; (b) The vector of $E_{a}$ under different voltage region; (c) The vectors of $A_{h}$ under different voltage region.

\subsection{Optimal Support Vector Machine Parameter Determination}

There are two key steps needed to properly build the SVM model: determination of the kernel function and selection of its parameters. The main function of the kernel function is to implement the nonlinear mapping from the original input space to a high dimension space. By comparison, the RBF is employed in this paper as the kernel function and can be formulated as

$$
K\left(x_{i}, x_{i}\right)=e^{\left(-g\left\|x_{i}-x_{j}\right\|^{2}\right)}=e^{-\frac{\left\|x_{i}-x_{j}\right\|^{2}}{2 \sigma^{2}}},
$$

where $g=1 / 2 \sigma^{2}$ and denotes width of the RBF. $C$ and $g$ need to be optimized which are crucial to improve the estimation precision of the SVM algorithm. Variety of approaches can be applied to optimize them. Among all the manners, the grid search algorithm is an exhaustive searching algorithm, which needs to be guided by some performance metric and typically measured by the cross-validation $(\mathrm{CV})$ on the training set [32]. Among all the CV methods, the k-fold CV (k-CV) is the most popular CV candidate, and thus we select it to train the RVM model. More concretely, $C$ and $g$ are ensured within a certain range and the minimal square error (MSE) is employed to evaluate the selection for different $C$ and $g$ based on the k-CV calculation,

$$
M S E=\frac{1}{l} \sum_{i=1}^{l}\left(f\left(x_{i}\right)-y_{i}\right)^{2},
$$

where $f\left(x_{i}\right)$ is the estimated $\mathrm{SOH}, y_{i}$ is the actual value, and $l$ is the number of testing samples. Then, we modify the range of grid search based on the results of the previous step and repeat the previous step 
until a suitable $(C, g)$ is found with target of minimizing the MSE. The purpose of the SVM model established in this paper is not to memorize input training sets, but to estimate the unknown input samples precisely. Hence, evaluation of generalization error is extremely important. To sum up, the employed CV algorithm is capable of avoiding the occurrence of over-fitting with the acceptable computational labor and consequently can reduce the generalization error.

For comprehensively evaluating the predictive performance, a combination of evaluation metrics is commonly employed [26,33]. Here, MSE and the mean absolute relative error (MARE) are referred together to evaluate the performance of the proposed method from different aspects, as show in (7) and (8).

$$
\text { MARE }=\frac{1}{l} \sum_{i=1}^{l} \frac{\left|f\left(x_{i}\right)-y_{i}\right|}{y_{i}} \times 100 \% .
$$

By giving relatively higher weighting to large errors, MSE is an appropriate index for avoiding larger deviations but cannot reflect the central tendency (average error) of the model. Mean absolute relative error is a natural tool for assessing average relative error of model, which can compensate for the shortcoming of MSE. Benefiting from these indexes, the predictive errors can be observed for deeper analysis. It is necessary to mention that the proposed method with regard to the SVM is realized based on LIBSVM toolbox under MATLAB/Simulink 2016a, which is developed by MathWorks with headquarters in Natick, Massachusetts, U.S.A [34]. The result validations are presented in the next section with detailed analysis.

\section{Predicted Results and Analysis}

To investigate the predictive accuracy of $\mathrm{SOH}$, experiment validations of four cells are conducted. Here, two different batteries Cell 1 and Cell 2 are adopted for the following discussion. The effective discharging and charging cycle number is 166 for Cell 1 and Cell 2. The SVM model is trained offline based on $60 \%$ charging cycle data, and the remaining charging data are applied for validation. In practice, all $\mathrm{CC}$ charging data are difficult to gain during the real-time $\mathrm{SOH}$ estimation because it is hardly possible to discharge the battery to its low threshold every time. Here, we also discuss the situation that only partial charging data can be obtained. In addition, another two cells, namely Cell 3 and Cell 4, are tested and their $\mathrm{SOH}$ estimation is conducted to validate the performance of the proposed algorithm.

\subsection{State of Health Estimation with All Charging Range}

For Cell 1 and Cell 2, the prediction results and errors of $\mathrm{SOH}$ estimation are presented in Figures 5-8. We can observe that, the proposed method based on the SVM algorithm performs precise estimation. As can be seen in Figures 6 and 8, most of error can be limited within 1.6\%. Moreover, for Cell 1, the maximum error, MSE and MARE are $0.98 \%, 6.5 \times 10^{-6}$ and $0.26 \%$, and for Cell 2, the maximum error, MSE and MARE are $1.87 \%, 9.6 \times 10^{-6}$ and $0.21 \%$, respectively. Hence, we can conclude that the proposed SVM model can achieve the reliable battery $\mathrm{SOH}$ estimation.

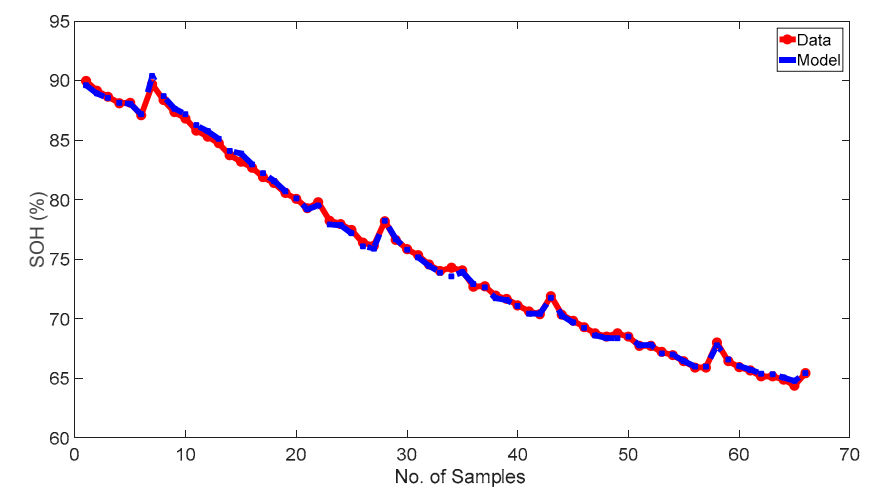

Figure 5. Results of SOH estimation by support vector machine (SVM) with Cell 1 testing samples. 


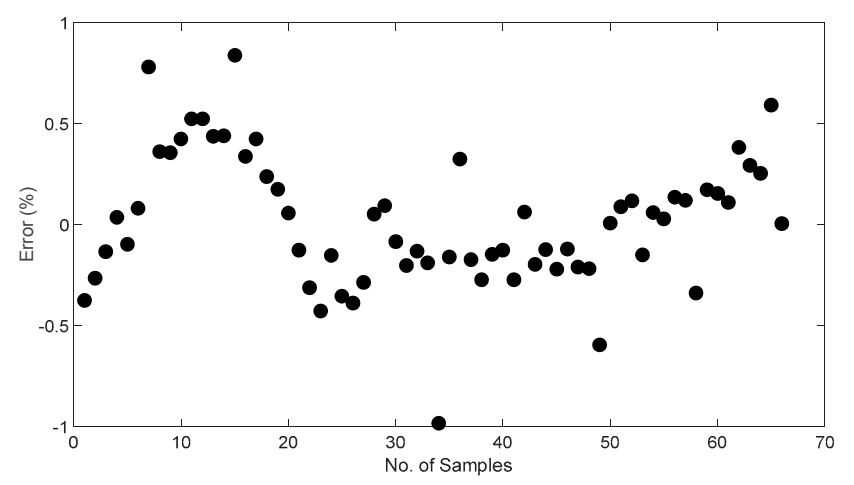

Figure 6. Error in SOH estimation by SVM with Cell 1 testing samples.

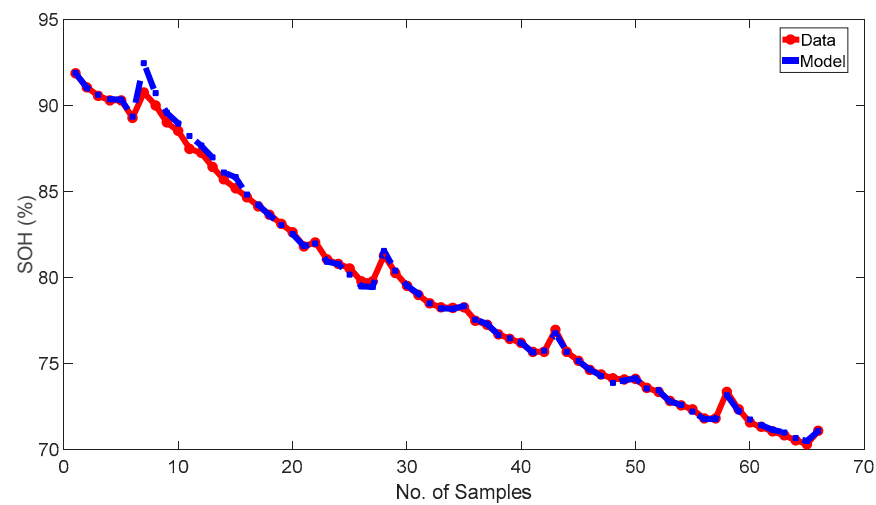

Figure 7. Results of SOH estimation by SVM with Cell 2 testing samples.

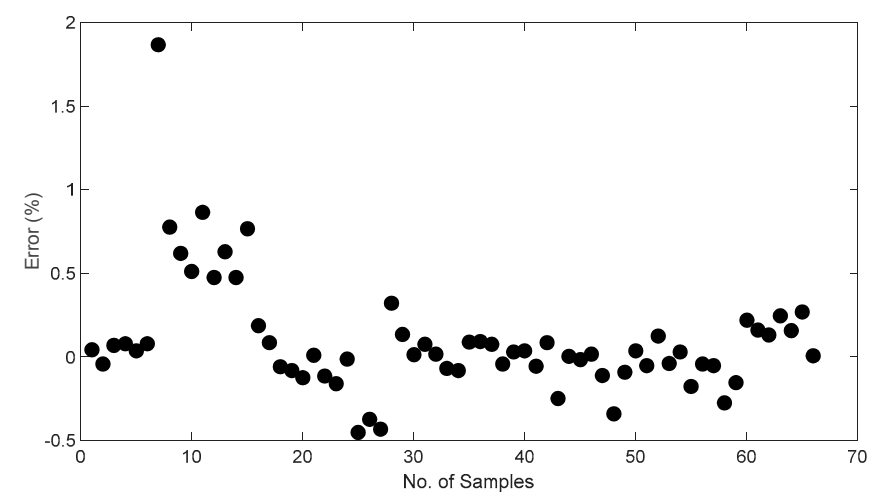

Figure 8. Error in SOH estimation by SVM with Cell 2 testing samples.

\subsection{State of Health Estimation with Partial Charging Range}

Here, the predicted results by the proposed method for different range of charging voltage are discussed. The MSE and MARE are selected as error indexes to evaluate the proposed model, and related results are shown in Table 3, and Figures 9 and 10, respectively. We selected the initial charging voltage from $3.75 \mathrm{~V}$ to $4.15 \mathrm{~V}$ with $0.05 \mathrm{~V}$ as the step interval. The data region ranges from $0.05 \mathrm{~V}$ to $0.55 \mathrm{~V}$. The results reveal that larger voltage range can bring more precise estimation results. As shown in Figures 9 and 10, the MSE and MARE for different voltage ranges of Cell 1 and Cell 2 are depicted. For both cells, different voltage region shows varied estimation precision. As shown in Figure 10 and Table 3, the voltage region $[3.75,4.20]$ validation exhibits minimum estimate error for Cell 1 . By comparison, the voltage region of $[3.95,4.00]$ is a suitable selection for Cell 1 , which optimally fits 
three standards mentioned above, and its MSE and MARE are $2.0 \times 10^{-5}$ and $0.41 \%$, respectively. Meanwhile, as shown in Figure 10 and Table 3, the voltage region of $[3.95,4.00]$ estimates SOH with MSE of $2.3 \times 10^{-5}$ and MARE of $0.36 \%$ for Cell 2, which are the optimal voltage region for Cell 2 . Moreover, compared with the MSE and MARE based on all charging voltage and current data, the estimation error can be accepted for Cell 1 and Cell 2 when applying the selected voltage region.

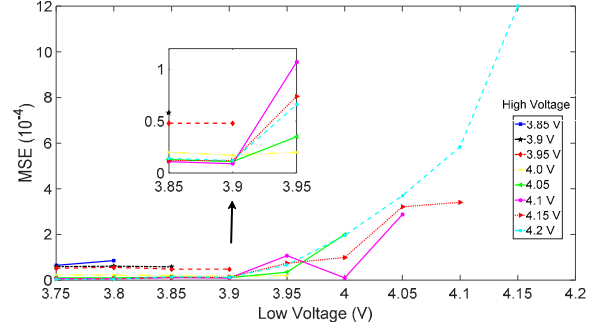

(a)

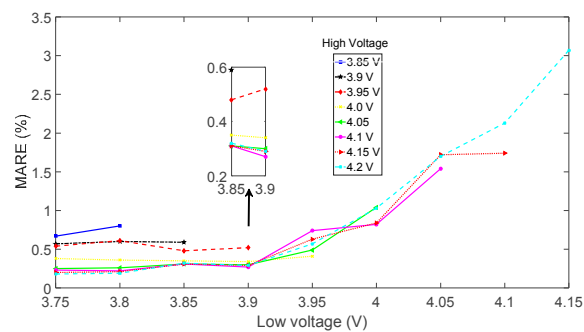

(b)

Figure 9. Error under different voltage region with Cell 1 testing samples: (a) MSE; and (b) MARE.

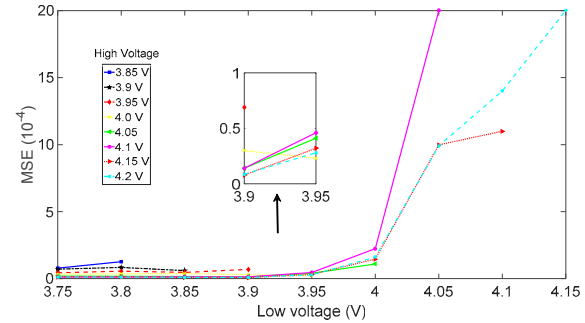

(a)

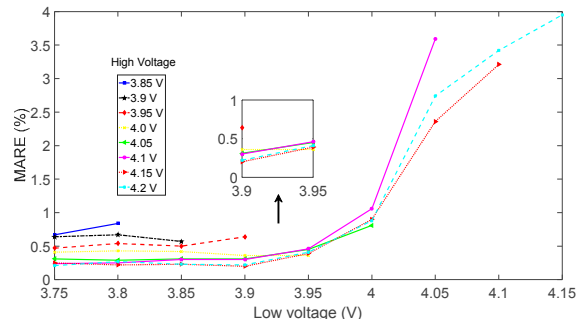

(b)

Figure 10. Error under different voltage region with Cell 2 testing samples: (a) MSE; and (b) MARE.

Table 3. Error performance under different voltage region.

\begin{tabular}{|c|c|c|c|c|c|c|c|c|c|}
\hline \multirow{2}{*}{$\begin{array}{l}\text { Voltage } \\
\text { Region }\end{array}$} & \multicolumn{2}{|c|}{ Cell 1} & \multicolumn{2}{|c|}{ Cell 2} & \multirow{2}{*}{$\begin{array}{l}\text { Voltage } \\
\text { Region }\end{array}$} & \multicolumn{2}{|c|}{ Cell 1} & \multicolumn{2}{|c|}{ Cell 2} \\
\hline & $\begin{array}{c}\text { MSE } \\
\left(10^{-4}\right)\end{array}$ & $\begin{array}{c}\text { MARE } \\
(\%)\end{array}$ & $\begin{array}{c}\text { MSE } \\
\left(10^{-4}\right)\end{array}$ & $\begin{array}{c}\text { MARE } \\
(\%)\end{array}$ & & $\begin{array}{c}\text { MSE } \\
\left(10^{-4}\right)\end{array}$ & $\begin{array}{c}\text { MARE } \\
(\%)\end{array}$ & $\begin{array}{l}\text { MSE } \\
\left(10^{-4}\right)\end{array}$ & $\begin{array}{c}\text { MARE } \\
(\%)\end{array}$ \\
\hline$[3.75,3.85]$ & 0.65 & 0.67 & 0.78 & 0.67 & {$[3.85,4.20]$} & 0.14 & 0.32 & 0.098 & 0.23 \\
\hline$[3.75,3.90]$ & 0.59 & 0.57 & 0.71 & 0.64 & {$[3.90,3.95]$} & 0.48 & 0.52 & 0.69 & 0.64 \\
\hline$[3.75,3.95]$ & 0.52 & 0.54 & 0.44 & 0.47 & {$[3.90,4.00]$} & 0.17 & 0.34 & 0.30 & 0.36 \\
\hline$[3.75,4.00]$ & 0.23 & 0.38 & 0.34 & 0.41 & {$[3.90,4.05]$} & 0.11 & 0.30 & 0.14 & 0.31 \\
\hline$[3.75,4.05]$ & 0.097 & 0.25 & 0.19 & 0.31 & {$[3.90,4.10]$} & 0.090 & 0.27 & 0.14 & 0.30 \\
\hline$[3.75,4.10]$ & 0.068 & 0.23 & 0.12 & 0.24 & {$[3.90,4.15]$} & 0.12 & 0.29 & 0.079 & 0.20 \\
\hline$[3.75,4.15]$ & 0.050 & 0.20 & 0.13 & 0.25 & {$[3.90,4.20]$} & 0.12 & 0.29 & 0.086 & 0.22 \\
\hline$[3.75,4.20]$ & 0.034 & 0.18 & 0.12 & 0.21 & {$[3.95,4.00]$} & 0.20 & 0.41 & 0.23 & 0.36 \\
\hline$[3.80,3.85]$ & 0.85 & 0.80 & 1.27 & 0.84 & {$[3.95,4.05]$} & 0.35 & 0.49 & 0.41 & 0.45 \\
\hline$[3.80,3.90]$ & 0.61 & 0.60 & 0.84 & 0.67 & {$[3.95,4.10]$} & 1.07 & 0.74 & 0.46 & 0.46 \\
\hline$[3.80,3.95]$ & 0.56 & 0.61 & 0.56 & 0.54 & {$[3.95,4.15]$} & 0.74 & 0.63 & 0.32 & 0.39 \\
\hline$[3.80,4.00]$ & 0.22 & 0.36 & 0.36 & 0.43 & {$[3.95,4.20]$} & 0.66 & 0.57 & 0.28 & 0.41 \\
\hline$[3.85,4.05]$ & 0.10 & 0.26 & 0.18 & 0.29 & {$[4.00,4.05]$} & 2.00 & 1.04 & 1.10 & 0.81 \\
\hline$[3.80,4.10]$ & 0.060 & 0.22 & 0.12 & 0.25 & {$[4.00,4.10]$} & 0.96 & 0.82 & 2.24 & 1.06 \\
\hline$[3.80,4.15]$ & 0.054 & 0.21 & 0.14 & 0.22 & {$[4.00,4.15]$} & 0.99 & 0.84 & 1.46 & 0.90 \\
\hline$[3.80,4.20]$ & 0.040 & 0.19 & 0.12 & 0.27 & {$[4.00,4.20]$} & 1.96 & 1.03 & 1.61 & 0.88 \\
\hline$[3.85,3.90]$ & 0.58 & 0.59 & 0.60 & 0.57 & {$[4.05,4.10]$} & 2.87 & 1.54 & 20 & 3.59 \\
\hline$[3.85,3.95]$ & 0.48 & 0.48 & 0.47 & 0.50 & {$[4.05,4.15]$} & 3.21 & 1.72 & 9.98 & 2.36 \\
\hline$[3.85,4.00]$ & 0.20 & 0.35 & 0.39 & 0.42 & {$[4.05,4.20]$} & 3.70 & 1.70 & 9.90 & 2.74 \\
\hline$[3.85,4.05]$ & 0.13 & 0.31 & 0.16 & 0.31 & {$[4.10,4.15]$} & 3.41 & 1.74 & 11 & 3.21 \\
\hline$[3.85,4.10]$ & 0.11 & 0.31 & 0.14 & 0.30 & {$[4.10,4.20]$} & 5.84 & 2.13 & 14 & 3.42 \\
\hline$[3.85,4.15]$ & 0.12 & 0.31 & 0.094 & 0.23 & {$[4.15,4.20]$} & 12 & 3.07 & 20 & 3.95 \\
\hline
\end{tabular}


Figures 11-14 compare the results of Cell 1 and Cell 2 under the selected voltage range, i.e., [3.95, 4.0]. In Figures 11 and 13, the proposed method can accurately estimate the $\mathrm{SOH}$ variation, of which the errors for both Cell 1 and Cell 2 can be limited to within 2\%. Thus, we can conclude that the prediction error maintains at the low error level and is applicable for practical application when partial charging data are available.

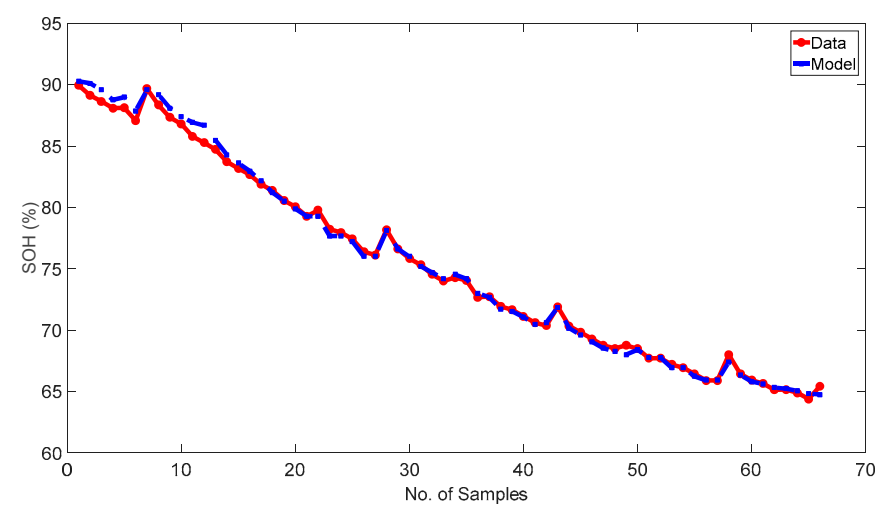

Figure 11. Results of $\mathrm{SOH}$ estimation with Cell 1 testing samples under the voltage region $[3.95,4.00]$.

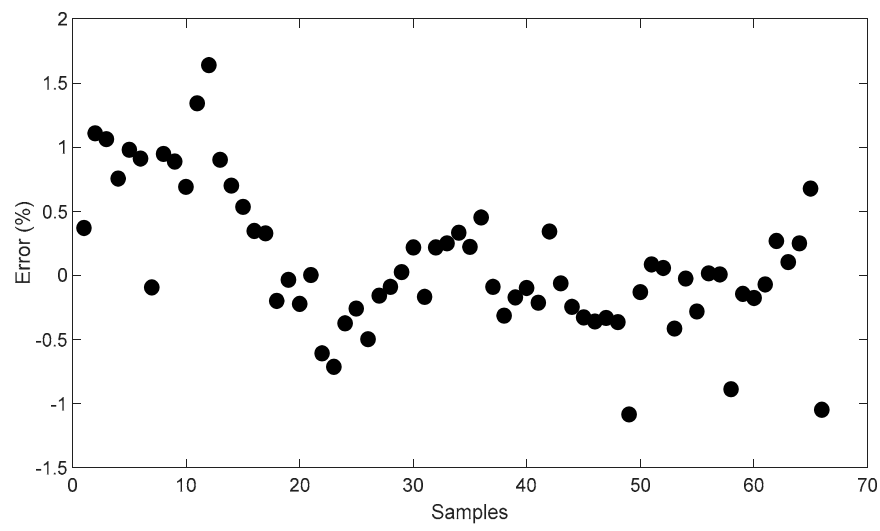

Figure 12. Error in SOH estimation with Cell 1 testing samples under the voltage region [3.95, 4.00].

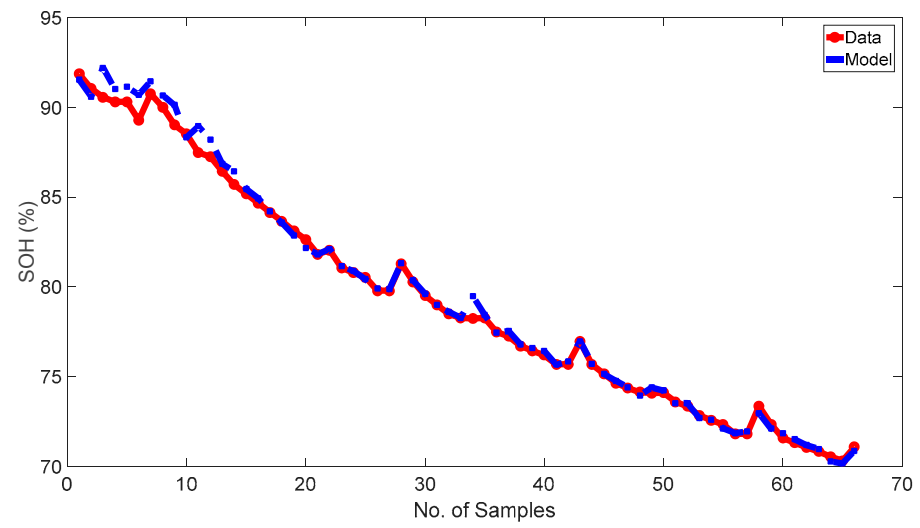

Figure 13. Results of SOH estimation with Cell 2 testing samples under the voltage region [3.95, 4.00]. 


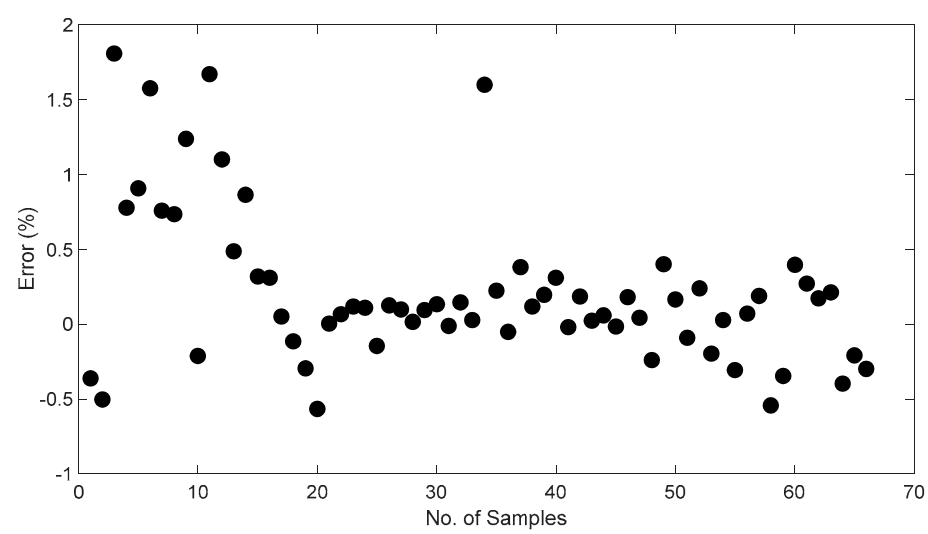

Figure 14. Error in $\mathrm{SOH}$ estimation with Cell 2 testing samples under the voltage region [3.95, 4.00].

\subsection{State of Health Estimation for Different Cells}

To extend validation for the proposed method, Cell 3 and Cell 4 are tested. The prediction results and errors of $\mathrm{SOH}$ estimation are presented in Figures 15 and 16 and Table 4. As shown in Figure 16 and Table 4, the proposed algorithm exhibits very small prediction errors. The MSE and MARE for two cells' SOH estimation are $0.45 \times 10^{-4}$ and $0.52 \times 10^{-4}$, and $0.93 \%$ and $0.88 \%$, respectively, proving the effectiveness when applying the algorithm to different cells.

Table 4. Error performance under voltage region $[3.95,4.00]$.

\begin{tabular}{ccc}
\hline Error & Cell 3 & Cell 4 \\
\hline MSE $\left(10^{-4}\right)$ & 0.45 & 0.93 \\
MARE $(\%)$ & 0.59 & 0.88 \\
\hline
\end{tabular}

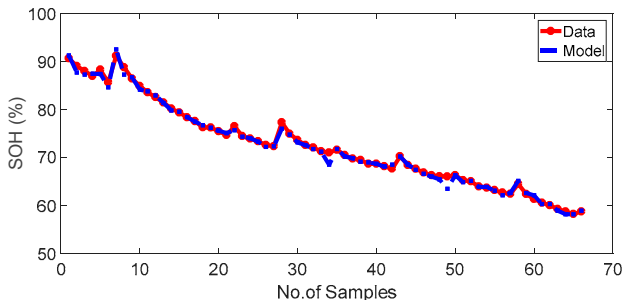

(a)

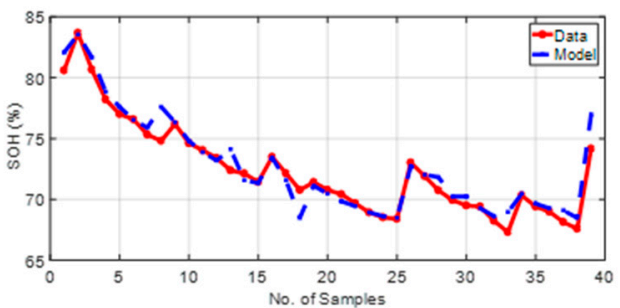

(b)

Figure 15. Results of $\mathrm{SOH}$ estimation with testing samples under the voltage region [3.95, 4.00]. (a) Cell 3; (b) Cell 4.

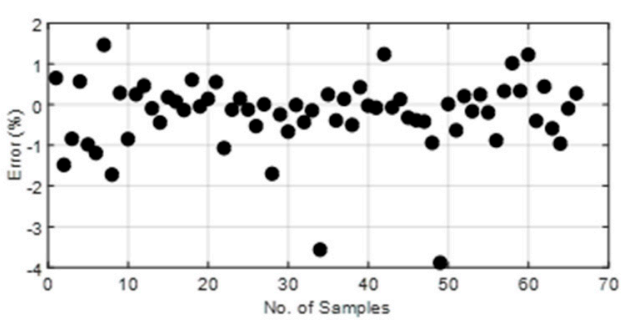

(a)

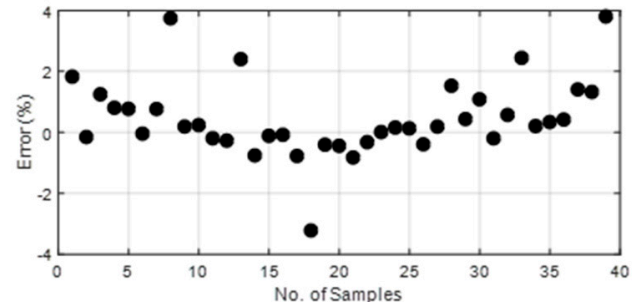

(b)

Figure 16. Error in $\mathrm{SOH}$ estimation with testing samples under the voltage region [3.95, 4.00]. (a) Cell 3; (b) Cell 4 . 


\section{Conclusions}

In this paper, a novel predictive method of $\mathrm{SOH}$ is addressed based on the charging data considering real application requirement. Three feature vectors, i.e., $E_{s}, A_{h}$, and $t$, are analyzed and chosen as characteristic variables, which are set to be the input of the proposed SVM model. The grid search method is employed to search the optimal kernel parameters of SVM model.

The partial charge data are considered to achieve the $\mathrm{SOH}$ estimation, where the voltage range of $3.95 \mathrm{~V}$ to $4.0 \mathrm{~V}$ is adopted given that it can satisfactorily obtain accurate predictions. The experiment results validate that the proposed method can efficiently predict the $\mathrm{SOH}$ online with less than $2 \%$ error. However, in actual application, sampling error, temperature and other factors could have an important impact on estimation precision and thus future study can be focused on them along with different voltage region validation.

In addition, battery pack $\mathrm{SOH}$ estimation should also be the research focus based on the proposed algorithm considering the computation intensity and data storage limitation. Furthermore, future research should focus on different lithium-ion batteries' $\mathrm{SOH}$ estimation, since they can exhibit different degradation mechanism and electrical performance.

Author Contributions: Z.C. and M.S. conceived this paper, carried out the battery experiment and completed the paper writing. R.X. discussed the SVM algorithm and revised the paper. J.S. conducted the SOH estimation and instruct the whole paper. X.S. provided some valuable suggestions.

Funding: This research received no external funding.

Acknowledgments: The work was supported by National Key R\&D Program of China (Grant No. 2018YFB0104000); National Natural Science Foundation of China (Grant No. 61763021); the Innovation Team Program of Kunming University of Science and Technology (Grant No. 14078368); and the Scientific Research Start-up Funding of Kunming University of Science and Technology (Grant No. 14078337). The authors would like to thank them for their support and assistance. Most importantly, the authors would like to thank the reviewers for their comments and suggestions.

Conflicts of Interest: The authors declare no conflict of interest.

\section{References}

1. Shen, J.-N.; He, Y.-J.; Ma, Z.-F.; Luo, H.-B.; Zhang, Z.-F. Online state of charge estimation of lithium-ion batteries: A moving horizon estimation approach. Chem. Eng. Sci. 2016, 154, 42-53. [CrossRef]

2. Wang, Y.; Zhang, C.; Chen, Z. A method for state-of-charge estimation of Li-ion batteries based on multi-model switching strategy. Appl. Energy 2015, 137, 427-434. [CrossRef]

3. Xiong, R.; Gong, X.; Mi, C.C.; Sun, F. A robust state-of-charge estimator for multiple types of lithium-ion batteries using adaptive extended Kalman filter. J. Power Sources 2013, 243, 805-816. [CrossRef]

4. Weng, C.; Feng, X.; Sun, J.; Peng, H. State-of-health monitoring of lithium-ion battery modules and packs via incremental capacity peak tracking. Appl. Energy 2016, 180, 360-368. [CrossRef]

5. Weng, C.; Feng, X.; Sun, J.; Ouyang, M.; Peng, H. Battery SOH Management Research in the US-China Clean Energy Research Center-Clean Vehicle Consortium. IFAC-PapersOnLine 2015, 48, 448-453. [CrossRef]

6. Moura, S.J.; Krstic, M.; Chaturvedi, N.A. Adaptive PDE observer for battery SOC/SOH estimation. Am. Soc. Mech. Eng. 2012, 101-110. [CrossRef]

7. Zhang, J.; Lee, J. A review on prognostics and health monitoring of Li-ion battery. J. Power Sources 2011, 196, 6007-6014. [CrossRef]

8. Rezvanizaniani, S.M.; Liu, Z.; Chen, Y.; Lee, J. Review and recent advances in battery health monitoring and prognostics technologies for electric vehicle (EV) safety and mobility. J. Power Sources 2014, 256, 110-124. [CrossRef]

9. Ng, S.S.Y.; Xing, Y.; Tsui, K.L. A naive Bayes model for robust remaining useful life prediction of lithium-ion battery. Appl. Energy 2014, 118, 114-123. [CrossRef]

10. Lyu, C.; Lai, Q.; Ge, T.; Yu, H.; Wang, L.; Ma, N. A lead-acid battery's remaining useful life prediction by using electrochemical model in the Particle Filtering framework. Energy 2017, 120, 975-984. [CrossRef]

11. Han, X.; Ouyang, M.; Lu, L.; Li, J. A comparative study of commercial lithium ion battery cycle life in electric vehicle: Capacity loss estimation. J. Power Sources 2014, 268, 658-669. [CrossRef] 
12. Li, Z.; Lu, L.; Ouyang, M.; Xiao, Y. Modeling the capacity degradation of $\mathrm{LiFePO}_{4} /$ graphite batteries based on stress coupling analysis. J. Power Sources 2011, 196, 9757-9766. [CrossRef]

13. Chen, Z.; Mi, C.C.; Fu, Y.; Xu, J.; Gong, X. Online battery state of health estimation based on Genetic Algorithm for electric and hybrid vehicle applications. J. Power Sources 2013, 240, 184-192. [CrossRef]

14. Mosallam, A.; Medjaher, K.; Zerhouni, N. Data-driven prognostic method based on Bayesian approaches for direct remaining useful life prediction. J. Intell. Manuf. 2014, 27, 1037-1048. [CrossRef]

15. Xiong, R.; Sun, F.; Chen, Z.; He, H. A data-driven multi-scale extended Kalman filtering based parameter and state estimation approach of lithium-ion olymer battery in electric vehicles. Appl. Energy 2014, 113, 463-476. [CrossRef]

16. Xiong, R.; Sun, F.; Gong, X.; Gao, C. A data-driven based adaptive state of charge estimator of lithium-ion polymer battery used in electric vehicles. Appl. Energy 2014, 113, 1421-1433. [CrossRef]

17. Zhou, Y.; Huang, M. Lithium-ion batteries remaining useful life prediction based on a mixture of empirical mode decomposition and ARIMA model. Microelectron. Reliabil. 2016, 65, 265-273. [CrossRef]

18. Awan, M.A.A. Remaining Useful life Prediction of Lithium-Ion Batteries Using Particle Filter Framework. Master's Thesis, Karlstad University, Faculty of Technology and Science, Karlstad, Sweden, 2014.

19. Dong, H.; Jin, X.; Lou, Y.; Wang, C. Lithium-ion battery state of health monitoring and remaining useful life prediction based on support vector regression-particle filter. J. Power Sources 2014, 271, 114-123. [CrossRef]

20. Wang, D.; Miao, Q.; Pecht, M. Prognostics of lithium-ion batteries based on relevance vectors and a conditional three-parameter capacity degradation model. J. Power Sources 2013, 239, 253-264. [CrossRef]

21. Liu, D.; Pang, J.; Zhou, J.; Peng, Y.; Pecht, M. Prognostics for state of health estimation of lithium-ion batteries based on combination Gaussian process functional regression. Microelectron. Reliabil. 2013, 53, 832-839. [CrossRef]

22. He, W.; Williard, N.; Osterman, M.; Pecht, M. Prognostics of lithium-ion batteries based on Dempster-Shafer theory and the Bayesian Monte Carlo method. J. Power Sources 2011, 196, 10314-10321. [CrossRef]

23. Michel, P.H.; Heiries, V. Book An Adaptive Sigma Point Kalman Filter Hybridized by Support Vector Machine Algorithm for Battery SoC and SoH Estimation. In Proceedings of the 2015 IEEE 81st Vehicular Technology Conference (VTC Spring), Glasgow, UK, 11-14 May 2015; pp. 1-7.

24. Patil, M.A.; Tagade, P.; Hariharan, K.S.; Kolake, S.M.; Song, T.; Yeo, T.; Doo, S. A novel multistage Support Vector Machine based approach for Li ion battery remaining useful life estimation. Appl. Energy 2015, 159, 285-297. [CrossRef]

25. Nuhic, A.; Terzimehic, T.; Soczka-Guth, T.; Buchholz, M.; Dietmayer, K. Health diagnosis and remaining useful life prognostics of lithium-ion batteries using data-driven methods. J. Power Sources 2013, 239, 680-688. [CrossRef]

26. Klass, V.; Behm, M.; Lindbergh, G. A support vector machine-based state-of-health estimation method for lithium-ion batteries under electric vehicle operation. J. Power Sources 2014, 270, 262-272. [CrossRef]

27. Weng, C.; Cui, Y.; Sun, J.; Peng, H. On-board state of health monitoring of lithium-ion batteries using incremental capacity analysis with support vector regression. J. Power Sources 2013, 235, 36-44. [CrossRef]

28. Saha, B.; Goebel, K.; Battery data set. NASA Ames Prognostics Data Repository. Available online: http:/ / ti.arc.nasa.gov/ project/ prognostic-data-repository (accessed on 22 May 2018).

29. Eker, Ö.; Camci, F.; Jennions, I.K. Major Challenges in Prognostics: Study on Benchmarking Prognostics Datasets. Available online: http:/ / dspace.lib.cranfield.ac.uk/handle/1826/9994 (accessed on 31 December 2012).

30. Ghamisi, P.; Couceiro, M.S.; Benediktsson, J.A. A Novel Feature Selection Approach Based on FODPSO and SVM. IEEE Trans. Geosci. Remote Sens. 2015, 53, 2935-2947. [CrossRef]

31. Wang, J.; Liu, P.; Hicks-Garner, J.; Sherman, E.; Soukiazian, S.; Verbrugge, M.; Tataria, H.; Musser, J.; Finamore, P. Cycle-life model for graphite-LiFePO 4 cells. J. Power Sources 2011, 196, 3942-3948. [CrossRef]

32. Duan, K.; Keerthi, S.S.; Poo, A.N. Evaluation of simple performance measures for tuning SVM hyperparameters. Neurocomputing 2003, 51, 41-59. [CrossRef]

33. Chai, T.; Draxler, R.R. Root Mean Square Error (RMSE) or Mean Absolute Error (MAE)? Geosci. Model Dev. Discus. 2014, 7, 1525-1534. [CrossRef]

34. Chang, C.-C.; Lin, C.-J. LIBSVM: A library for support vector machines. ACM Trans. Intell. Syst. Technol. 2007, 2. [CrossRef]

(C) 2018 by the authors. Licensee MDPI, Basel, Switzerland. This article is an open access article distributed under the terms and conditions of the Creative Commons Attribution (CC BY) license (http://creativecommons.org/licenses/by/4.0/). 